AIGO: a southern hemisphere detector for the worldwide array of ground-based interferometric gravitational wave detectors

This article has been downloaded from IOPscience. Please scroll down to see the full text article.

2010 Class. Quantum Grav. 27084005

(http://iopscience.iop.org/0264-9381/27/8/084005)

View the table of contents for this issue, or go to the journal homepage for more

Download details:

IP Address: 131.251.133.27

The article was downloaded on 04/04/2012 at 12:42

Please note that terms and conditions apply. 


\title{
AIGO: a southern hemisphere detector for the worldwide array of ground-based interferometric gravitational wave detectors
}

\author{
P Barriga ${ }^{1}$, D G Blair ${ }^{1}$, D Coward ${ }^{1}$, J Davidson ${ }^{1}$, J-C Dumas ${ }^{1}$, \\ E Howell ${ }^{1}$, L Ju ${ }^{1}$, L Wen ${ }^{1}, C$ Zhao ${ }^{1}$, D E McClelland ${ }^{2}, S$ M Scott $^{2}$, \\ B J J Slagmolen ${ }^{2}$, R Inta ${ }^{2}$, J Munch ${ }^{3}$, D J Ottaway ${ }^{3}$, P Veitch ${ }^{3}$,

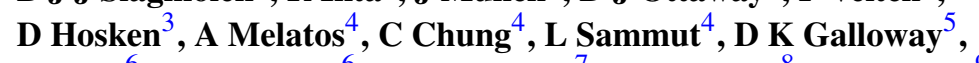 \\ J Marx ${ }^{6}$, S Whitcomb ${ }^{6}$, D Shoemaker ${ }^{7}$, S A Hughes ${ }^{8}$, D H Reitze ${ }^{9}$, \\ B R Iyer ${ }^{10}$, S V Dhurandhar ${ }^{11}$, T Souradeep ${ }^{11}$, C S Unnikrishnan ${ }^{12}$, \\ G Rajalakshmi $^{12}, \mathbf{C}$ N Man ${ }^{13}$, A Heidmann ${ }^{14}$, P-F Cohadon ${ }^{14}$,

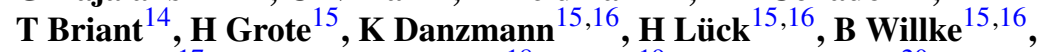 \\ K A Strain ${ }^{17}, \mathbf{B}$ S Sathyaprakash ${ }^{18}, \mathbf{J ~ C a o}{ }^{19}$, Y-K E Cheung ${ }^{20}$ and \\ Y Zhang ${ }^{21}$ \\ ${ }^{1}$ School of Physics, The University of Western Australia, Crawley, WA 6009, Australia \\ 2 Department of Physics, Faculty of Science, Australian National University, Canberra, \\ ACT 0200, Australia \\ ${ }^{3}$ Department of Physics, University of Adelaide, Adelaide, SA 5005, Australia \\ ${ }^{4}$ School of Physics University of Melbourne, Parkville, Vic 3010, Australia \\ ${ }^{5}$ School of Mathematical Sciences, Monash University, Vic 3800, Australia \\ ${ }^{6}$ LIGO_California Institute of Technology, Pasadena, CA 91125, USA \\ ${ }^{7}$ LIGO-Massachusetts Institute of Technology, Cambridge, MA 02139, USA \\ ${ }^{8}$ Department of Physics, Massachusetts Institute of Technology and MIT Kavli Institute, \\ Cambridge, MA 02139, USA \\ ${ }^{9}$ University of Florida, Gainesville, FL 32611, USA \\ ${ }^{10}$ Raman Research Institute, C. V. Raman Avenue, Sadashivanagar, Bangalore 560080 , India \\ ${ }^{11}$ Inter-University Centre for Astronomy and Astrophysics, Post Bag 4, Ganeshkhind, \\ Pune 411007 , India \\ 12 Gravitation Group, Tata Institute of Fundamental Research, Homi Bhabha Road, \\ Mumbai 400 005, India \\ ${ }^{13}$ Université Nice Sophia-Antipolis, CNRS, Observatoire Côte d'Azur, BP 4229, \\ 06304 Nice Cedex 4, France \\ ${ }^{14}$ Laboratoire Kastler Brossel, CNRS, Ecole Normale Supérieure and Université P. et M. Curie, \\ Case 74, 4 place Jussieu, F75252 Paris Cedex 05, France \\ 15 Albert-Einstein-Institut, Max-Planck-Institut für Gravitationsphysik, D-30167 Hannover, \\ Germany \\ ${ }^{16}$ Leibniz Universität Hannover, D-30167 Hannover, Germany \\ ${ }^{17}$ University of Glasgow, Glasgow G12 8QQ, UK \\ 18 Cardiff University, Cardiff CF24 3AA, UK \\ 19 Tsinghua University, Beijing 100084, People's Republic of China \\ 20 Department of Physics, Nanjing University, 22 Hankou Road, Jiangsu 210098, \\ People's Republic of China \\ 21 Department of Physics, University of Science and Technology of China, Hefei, Anhui 230026, \\ People's Republic of China \\ E-mail: pbarriga@cyllene.uwa.edu.au
}


Received 10 November 2009, in final form 21 December 2009

Published 6 April 2010

Online at stacks.iop.org/CQG/27/084005

\begin{abstract}
This paper describes the proposed AIGO detector for the worldwide array of interferometric gravitational wave detectors. The first part of the paper summarizes the benefits that AIGO provides to the worldwide array of detectors. The second part gives a technical description of the detector, which will follow closely the Advanced LIGO design. Possible technical variations in the design are discussed.
\end{abstract}

PACS numbers: 04.80.Nn, 04.30.Tv, 07.05.Dz, 07.10.Fq, 07.30.Bx

(Some figures in this article are in colour only in the electronic version)

\title{
1. Introduction: science benefits of AIGO
}

An individual gravitational wave $(\mathrm{GW})$ detector is almost omni-directional with a wide antenna pattern and poor angular resolution. In order to undertake GW astronomy and astrophysics, a network of detectors is indispensable. The existing network consisting of the two LIGO sites [1], Virgo [2] and GEO600 [3] has poor angular resolution along directions perpendicular to the line connecting the USA and Europe, and correspondingly poor polarization information. Limited information about the wave strength in the two polarizations will leave many questions about the sources unanswered, and underlines the case for extension of the network. The addition of a southern hemisphere detector maximizes the network baseline, improving sensitivity and sky coverage and enabling astrophysical studies.

Advanced LIGO [4], Advanced Virgo [5] and GEO-HF [6] are upgrades of the initial ground-based interferometric GW detectors. They are currently under construction. To enhance this network new detectors are proposed: LCGT [7] in Japan, AIGO in Australia and IndIGO a possible detector in India (figure 1). These advanced detectors are designed to have improved low frequency performance and lower shot noise, leading to amplitude sensitivity about 10 times better than existing detectors, enabling them to monitor a volume of the universe 1000 times larger than current detectors. They are expected to enable frequent detection of GW sources in the $10 \mathrm{~Hz}-10 \mathrm{kHz}$ range. Localization and identification of sources, plus full extraction of polarization data from gravitational waveforms and joint GW-electromagnetic (EM) studies will allow general relativity to be probed in the strong field regime.

\subsection{Angular resolution and source localization}

The addition of another detector, well out of the plane of the existing network, enables improved angular resolution for GW sources that would otherwise be poorly resolved.

For transient sources, such as short bursts and binary inspirals, the network directional sensitivity is determined by comparison of the signal phase at widely spaced detectors. Thus a fourth detector, placed well out of the plane formed by the existing three, will allow the number of sources that can be located to within a single galaxy to be increased by a factor of 3 [8].

AIGO improves the ability to determine, from GW detection alone, the location of the sources on the sky. The coherent analysis of signals from the array allows the network to have 


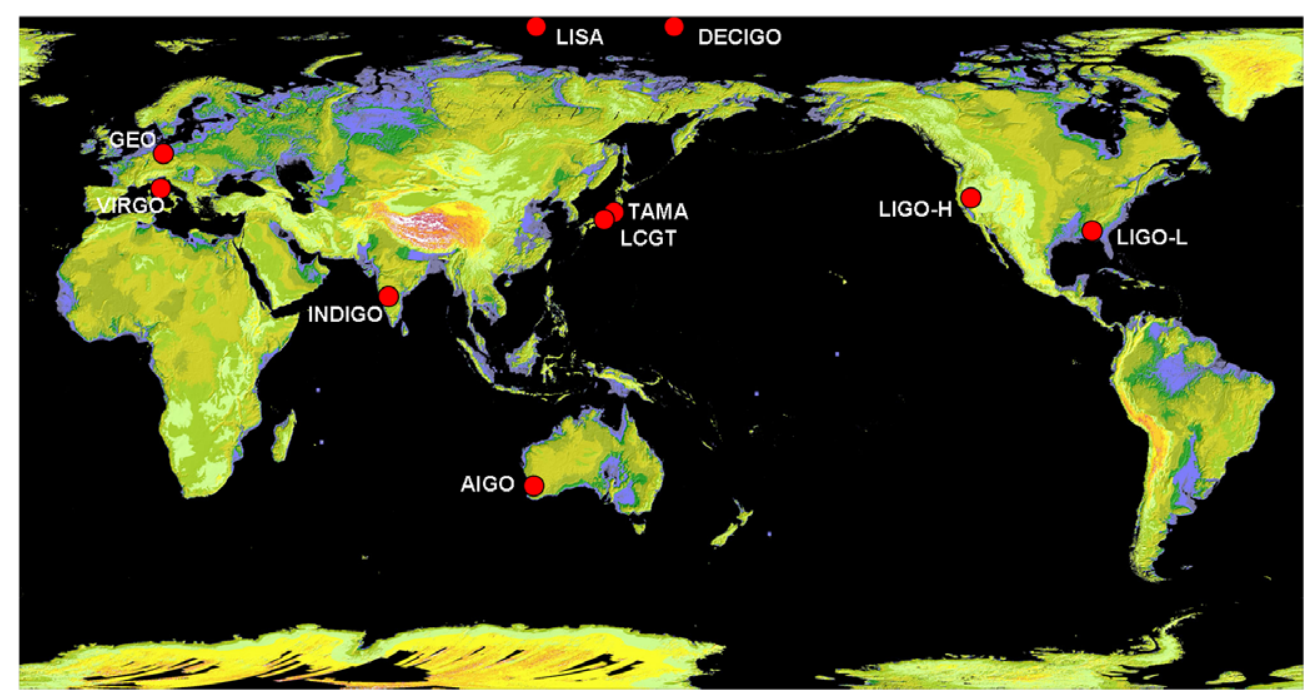

Figure 1. Location of existing and proposed interferometric gravitational wave detectors including the proposed space interferometers LISA and DECIGO.

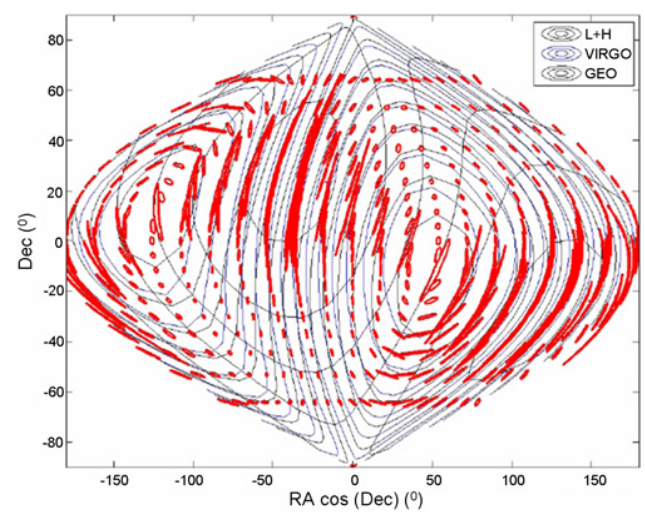

(a)

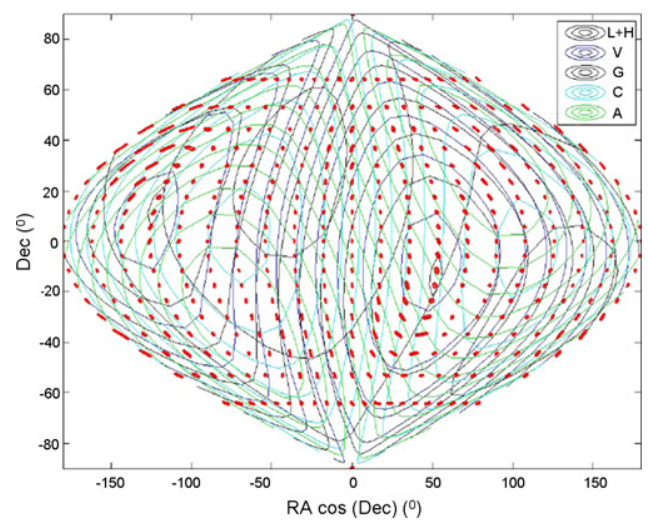

(b)

Figure 2. Angular area maps for a world network of interferometric GW detectors. (a) The antenna pattern for an array including LIGO Livingston (L), LIGO Hanford (H), Virgo (V) and GEO600 (G). (b) The antenna pattern when LCGT (C) and AIGO (A) are included. The angular uncertainty is shown as red ellipsoids in the sky, with a clear reduction of the uncertainty by adding LCGT and AIGO to the GW network [8]. AIGO reduces the error ellipses from typically about $1.5 \mathrm{deg} \times 8 \mathrm{deg}$ to a much smaller uncertainty of $1 \mathrm{deg} \times 1 \mathrm{deg}$.

diffraction limited resolution, where, as with VLBI radio astronomy, the angular resolution is set by the ratio of the signal wavelength to the product of the projected detector spacing in a direction perpendicular to the source and the signal-to-noise ratio. Detailed calculations by Wen et al [8] indicate that inclusion of AIGO would improve the international network's ability to localize sources. These calculations assume that the sensitivity of AIGO is comparable to the Advanced LIGO one. Figure 2 shows a significant improvement in the angular uncertainty on the sky when adding AIGO to the network of GW detectors. The increase in maximum baseline improves the angular resolution in almost all sky directions. It also reduces the 


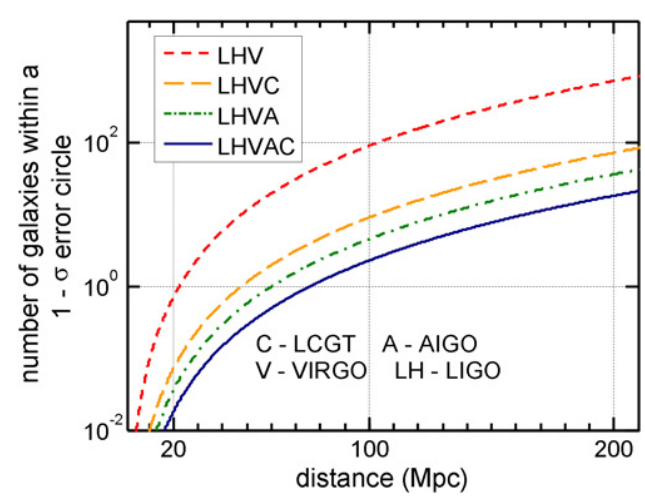

Figure 3. The average number of galaxies expected within a $1-\sigma$ error ellipse for different gravitational wave detector arrays, based on the angular resolution of each array for each sky direction. The figure shows that the number of galaxies per error ellipse is reduced from almost 200 for the LIGO-Virgo array, to less than 10 for LIGO-Virgo-AIGO. For a single additional detector, AIGO gives the greatest benefit, but the best array contains AIGO and LCGT.

number of galaxies within an average error ellipse by one order of magnitude as figure 3 shows; the array is further improved if LCGT is added. We note that since the angular resolution is inversely proportional to the frequency of the source, the resolution will depend on the source type.

\subsection{Gravitational waves polarization}

The primary goal of developing the science of GW astronomy requires the measurement of both GW polarizations, as this increases the potential to extract astrophysical information. To extract the two orthogonal polarizations $\left(h_{+}\right.$and $\left.h_{\times}\right)$of the the incident wave, a network with detectors of different orientation in three dimensions is required.

The overlap of the individual antenna patterns spreads over the spherical surface of the Earth creating a non-uniform global antenna pattern. This complex network antenna pattern rotates relative to a galaxy distribution pattern. The ability of the network antenna to resolve individual host galaxies will depend on the instantaneous coincidence of the antenna pattern with the galaxy distribution pattern.

Consider, for example, waves from a binary coalescence. Advanced detectors are expected to detect $\sim 40$ events per year. In this case, the amplitude ratio of the two GW polarizations encodes the inclination of the plane of the binary orbit with respect to the line of sight from the Earth. Once the orbital inclination is defined, the frequency evolution of the gravity wave signal contains a complete description of the system, and the observed amplitude therefore encodes the distance of the source. It is this remarkable property of GW signals that enables them to be powerful cosmological probes. Adding an Australian detector to the network augments its capability to measure polarizations simply due to its orientation on the nearly spherical surface of the Earth. Thus AIGO enables the network to obtain two to three times improved precision for distance estimation of sources [9].

\subsection{Gravitational waves and electromagnetic waves}

The correlation of EM sources with GW signals provides enormous science benefits. It is possible to search for EM transients correlated with candidate GW events that alone would have insufficient signal-to-noise ratio to be able to claim detection. Thus, instead of restricting 
candidate detection to high signal-to-noise ratio events, corresponding to say one false positive detection in 50 years, one can work with a reduced signal-to-noise where the false positive rate is say one per day. If the angular resolution is adequate, then EM detectors can search the error ellipse for a coincident afterglow. An EM source spatially and temporally correlated with a GW candidate is extremely unlikely. Therefore low signal-to-noise ratio GW events can translate into highly significant detections of GW. In this way EM correlation enables an enhancement in the sensitivity and the horizon distance for the GW detector array, corresponding to a substantially improved event rate.

If a detected GW burst or inspiral chirp of GW is accompanied by an EM signal such as a gamma-ray burst, then a comparison of the arrival times of the two bursts will give an immediate, high-precision measurement of the speed of GW relative to that of light. Such a test depends crucially on having sufficient angular resolution for the GW signal to identify reliably that the EM pulse came from the same source.

For binary black hole inspirals, the luminosity distance can be determined from the GW inspiral event, independent of the red shift determined from observation of the host galaxy. This allows a powerful independent probe of the Hubble law [10], cosmological acceleration and the equation of state of dark energy [9].

Optical telescopes distributed around the world can potentially respond to GW triggers and search the relevant sky directions. A $1 \mathrm{~m}$ fast response robotic telescope has been installed at the proposed AIGO site. It is primed to respond to external triggers robotically and has already observed gamma-ray burst afterglows as faint as apparent magnitude $\sim 23$ [11].

\subsection{Noise performance}

Broadband stationary noise in a network of detectors is reduced as the square root of the number of detectors. While this factor is not large $(\sim 25 \%)$, it has a much larger effect on the number of detectable sources. This number depends on the volume of the accessible universe, which increases as the cube of the detector strain sensitivity. Therefore, the addition of a southern hemisphere detector of sensitivity comparable to the advanced detectors is expected to double the number of detectable sources.

All detectors experience some periods of downtime (due to strong seismic events for example); an additional detector has a significant effect on reducing array downtime, thereby ensuring continuous monitoring of rare events. For non-stationary noise, a larger network has the benefit of being much better at rejecting spurious signals. Such signals must mimic a GW passing through the network by arriving at each detector at a time and with appropriate amplitude to be consistent with a real GW signal. The probability of such a false alarm reduces as the power of the number of detectors, so the addition of a single detector reduces this probability by a factor of 10-100 depending on the types of signal.

The improvements that the addition of AIGO provides to the GW network enhances the knowledge we can gain about the GW sources. For some sources, such as core collapse supernovae, the waves are likely to be poorly understood prior to $\mathrm{GW}$ observations. While signals from black hole binary mergers are likely to be only moderately well understood, waves from coalescing binaries prior to merger are well understood. In the not-wellunderstood regime one must use the observed waves to solve an 'inverse' problem and obtain an understanding of the dynamics of the source. Gürsel and Tinto [12] developed methods for performing the 'inverse' problem, and examined how well it could be implemented using detectors located in North America, Europe and Australia. Their work demonstrates that the addition of AIGO to the network improves the reconstruction of such waves. In the other extreme of a well-understood system, the signal can be used to define the source distance and location on the sky. 


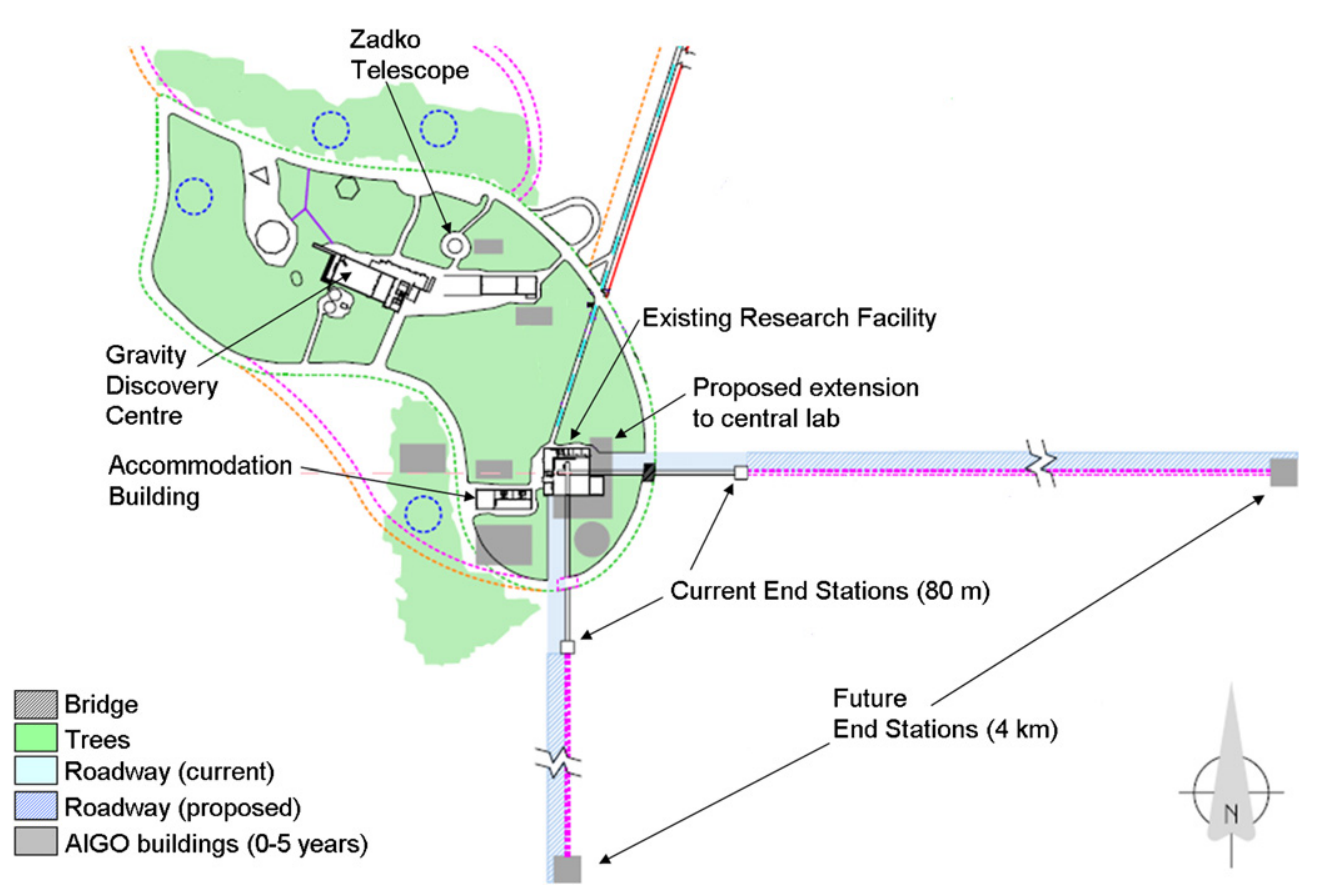

Figure 4. Proposed AIGO site at Wallingup plain $80 \mathrm{~km}$ north of Perth in Western Australia.

\section{AIGO: technical description}

It is proposed that AIGO be located on Wallingup plain, the site of the current Gingin research facility. The site consists of a flat $50 \mathrm{~km}^{2}$ area of deep silica sand. This provides excellent attenuation of seismic waves in the $4-20 \mathrm{~Hz}$ frequency band [13] suitable for a sensitive instrument like an interferometric GW detector. The nearby Gravity Discovery Centre, a public education centre, was designed to be far enough away to prevent seismic disturbance from visitor activity. Figure 4 shows a diagram of the proposed site for AIGO. The interferometer arms will be located on flat limestone roadways which can be created at low cost due to the flatness, stability and ease of construction in this landscape.

Here, we summarize the main features of AIGO, which is based on the design of Advanced LIGO. As a consequence the predicted sensitivity of AIGO is also similar to Advanced LIGO, which is presented in figure 5 .

\subsection{Optical configuration}

The proposed AIGO design is a dual recycled Michelson interferometer with Fabry-Perot arm cavities. The arm cavities are defined by the partially reflecting input test mass (ITM) and the high reflectivity end test mass (ETM). By placing a partially reflecting mirror at the input of the interferometer before the beam splitter, the bright fringe (which corresponds to the common mode strains of the two arms) will be reflected back into the interferometer arms. This power recycling technique increases the circulating power in the arm cavities without affecting the frequency response of the interferometer [14].

The first generation of interferometric GW detectors (LIGO and Virgo) used marginally stable power recycling cavities in an essentially flat/flat configuration. These cavities did not 


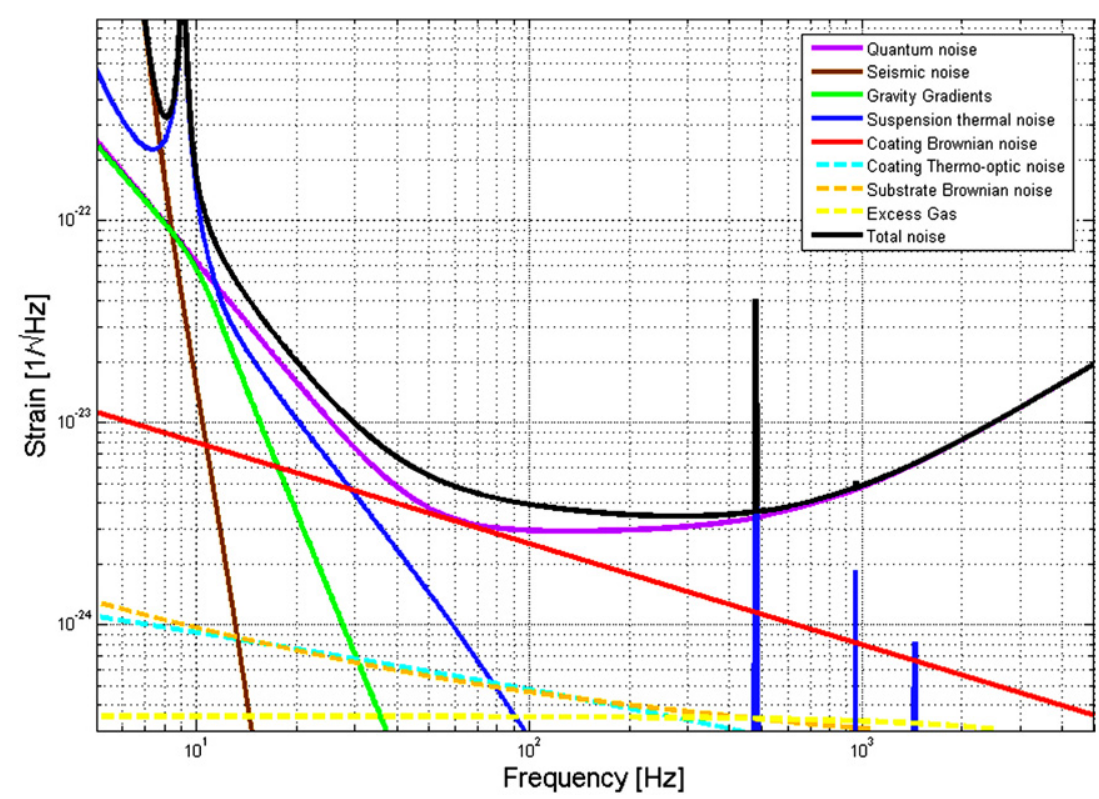

Figure 5. Advanced LIGO modelled noise budget. Due to the similarity in design it is expected that AIGO will have a similar noise budget as Advanced LIGO. The figure shows the different noise sources for non-detuned signal recycling cavity as modelled with the GWINC-v2 software package. It also assumes an input laser power of $125 \mathrm{~W}$.

confine the spatial modes of the RF sidebands which led to significant spatial mode mismatch between them and the carrier. Also marginally stable signal recycling cavities will reduce the amplitude of the GW sidebands by resonantly enhancing the scattering of light into higher order spatial modes. As a consequence, stable recycling cavities are an integral part of the optical design of the next generation of interferometric GW detectors $[15,16]$. Figure 6 shows the proposed optical layout for AIGO, including an input mode cleaner (IMC), a power recycling cavity (PRC), two arm cavities, a signal recycling cavity (SRC) and an output mode cleaner (OMC). The IMC has been thoroughly studied $[17,18]$ and the current design includes a suspended IMC [19]; however, an optical fibre mode cleaner is being studied by research institutes in France and could be included in the AIGO design [20, 21].

Due to the higher circulating power in advanced detectors, test masses that form the Fabry-Perot arms are made up of high purity fused silica to reduce thermal effects. To control these effects a fused silica compensation plate will be installed behind each ITM inside the PRC; sensing will be done using off-axis Hartmann sensors. This configuration had been successfully tested at the Gingin test facility [22]. Test masses will be heavier $(\sim 40 \mathrm{~kg})$ in order to reduce the effects of radiation pressure. Multi-layer dielectric coatings are being developed to reduce the related thermal noise [23]. Large spot sizes are needed in order to maximize the averaging over the thermal fluctuations in the test masses and thereby reduce the test mass thermal noise. At the same time it is necessary to keep the fundamental mode diffraction losses sufficiently low (normally below $1 \mathrm{ppm}$ per round trip).

\subsection{Sensing and control}

All the optical cavities mentioned above need to be kept locked at all times in order to be able to detect GW. A sophisticated sensing and control system is then required. Sensing and 


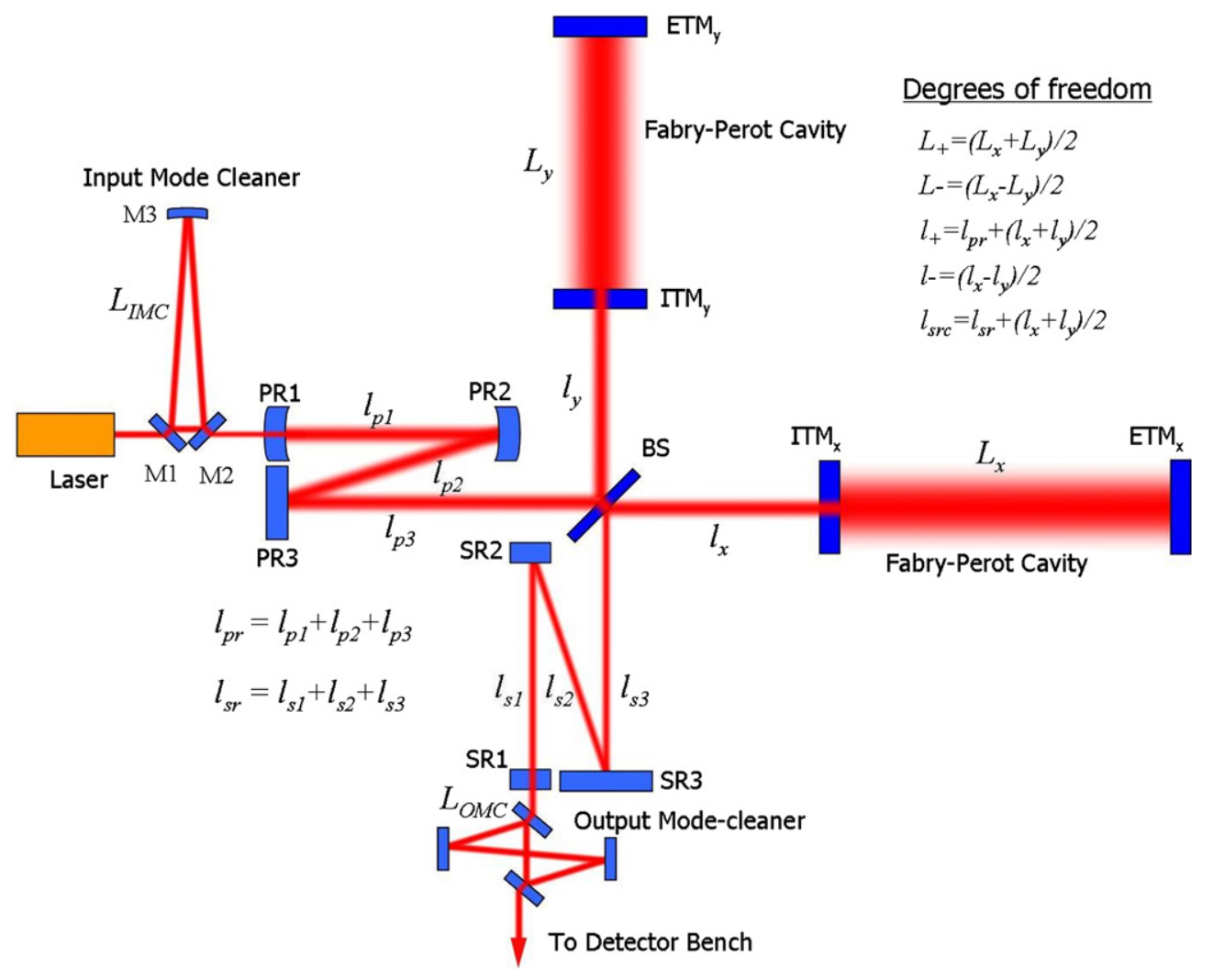

Figure 6. Dual recycled advanced interferometric GW detector proposed for AIGO. The figure shows stable recycling cavities and length degrees of freedom, including input mode cleaner (IMC) and output mode cleaner (OMC). The power recycling mirror (PRM), beam splitter (BS), both input test masses (ITMx and ITMy), and both end test masses (ETMx and ETMy). It shows stable power recycling cavity (PRC) formed by the power recycling mirrors (PR1, PR2 and PR3) and both input test masses passing through the BS. Also a stable signal recycling cavity (SRC) formed by the signal recycling mirrors (SR1, SR2 and SR3) and both ITMs. It also shows the different degrees of freedom that need to be controlled.

control ports of the interferometer will be equipped with wavefront sensors (WFS) sensitive to the RF sidebands; optical levers are considered for angular control of the core optics and CCD cameras for beam positioning on the high reflectivity surface of the core optics. In addition, the transmitted beam of each optic will be monitored by a quad-photo-detector (QPD) sensitive to the carrier light [24].

In principle two radio frequency (RF) sidebands are enough to control all 5 length degrees of freedom of an advanced dual recycled interferometer. These two RF modulation frequencies ( $f_{1}$ and $f_{2}$ ) make the use of double and/or differential demodulation techniques possible. The two RF sidebands allow us to obtain length signals using single demodulation at $f_{1}$ and $f_{2}$ or differential demodulation at $f_{1} \pm f_{2}$, and double demodulation at a combination of $f_{1}-f_{2}$ and $f_{1}+f_{2}$ where the signal is produced by the beat between the two RF sidebands, equivalent to $\left(f_{1} \times f_{2}\right)[25]$.

The input signals for the sensing and control systems come from the different sensors installed around the interferometer. Tuned signals that are band-pass filtered before being 
synchronously demodulated are used for detection of the various degrees of freedom. Each of these signals is fed to an input matrix where each length degree of freedom can be calculated. These are the differential arm length $\left(L_{-}\right)$, Michelson length $\left(l_{-}\right)$, common arm length $\left(L_{+}\right)$, power recycling cavity length $\left(l_{+}\right)$and signal recycling cavity length $\left(l_{s r c}\right)$. Figure 6 shows their definitions in the advanced dual recycled interferometer configuration proposed for AIGO.

The addition of a SRC and its operation in a detuned configuration will introduce an imbalance on the control sidebands reducing the sensitivity of the interferometer [26]. By introducing a very little offset in the Michelson arms (picometre scale) a small amount of carrier light will appear at the output port. This small fraction of carrier light is then used as the local oscillator necessary to demodulate the output signal and read the GW signal. This technique known as direct-conversion (DC) readout was proposed in 2000 by Fritschel [27]; it is being tested in Enhanced LIGO and it will be implemented in the advanced detectors (Advanced LIGO, Advanced Virgo and GEO-HF). It has several technical advantages, including the increase of signal to shot noise ratio by eliminating the vacuum fluctuations at twice the frequency of the modulation [28]. DC readout also allows for the addition of a short $(\sim 1 \mathrm{~m})$ OMC that rejects all but the fundamental mode component of the contrast defect [29]. In future configurations the OMC could provide an excellent reference for aligning the injection of squeezed light [30].

\subsection{Vibration isolation}

Advanced LIGO has been chosen to implement a 'stiff' pre-isolation technique based on hydraulic systems distributed at each corner of the vacuum chamber, complemented by a two-stage six-DOF active isolator in vacuum [31]. These provide vertical and horizontal preisolation for the whole chamber combining geophones and seismometers with high resonant frequencies in a high-bandwidth control loop. A four-stage pendulum test-mass suspension provides significant additional isolation in the GW band.

A different approach in terms of pre-isolation has been taken by other groups adopting a 'soft' design [32-34]. Primarily based on very low resonant frequencies, these are achieved with different damping solutions based on anti-spring designs and electromagnetic coupling, complemented with low bandwidth feedback control loops. A vibration isolation system based on multiple cascaded stages, see figure 7(a), has been developed by UWA. The design includes two stages of horizontal pre-isolation, and one stage of vertical pre-isolation with resonant frequencies $\sim 100 \mathrm{mHz}$. The nested structure facilitates a compact design and enables horizontal pre-isolation stages to be configured to create a super-spring configuration [35], where active feedback can enable performance close to the limit set by seismic tilt coupling. The pre-isolation stages are combined with multistage self-damped pendulums, where Euler springs are used to obtain vertical normal modes well matched to the pendulum frequencies [36]. In the current system the test mass is attached to the control mass through a niobium flexure suspension that minimize internal modes and provides a high $Q$-factor [37]. This could be replaced with a fused silica suspension if required. While the vibration isolation system is mainly of passive design, some feedback is required for low frequency control, such as alignment and drift corrections, and damping of normal modes [38]. A pair of full scale isolators are in operation at the Gingin facility and have been used to lock a $72 \mathrm{~m}$ suspended cavity with finesse $\sim 750$ [39]. Figure $7(b)$ shows the measured cavity displacement for the UWA system and the expected displacement once the system has been upgraded with a super-spring configuration. Mostly passive, and relatively low cost, it needs to be confirmed through careful review, that it is compatible with the Advanced LIGO baseline design chosen for AIGO. 


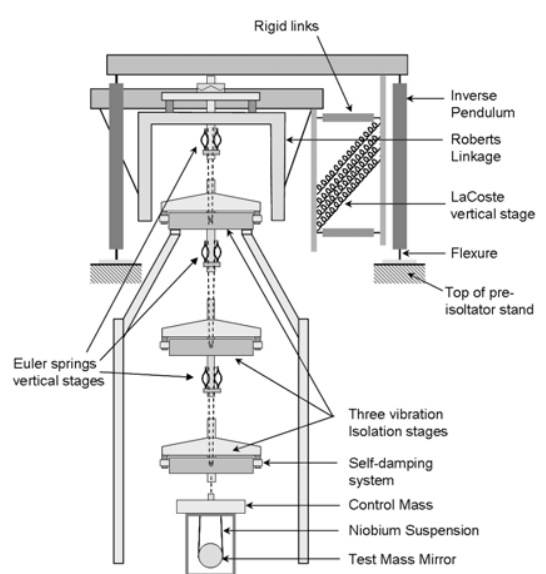

(a)

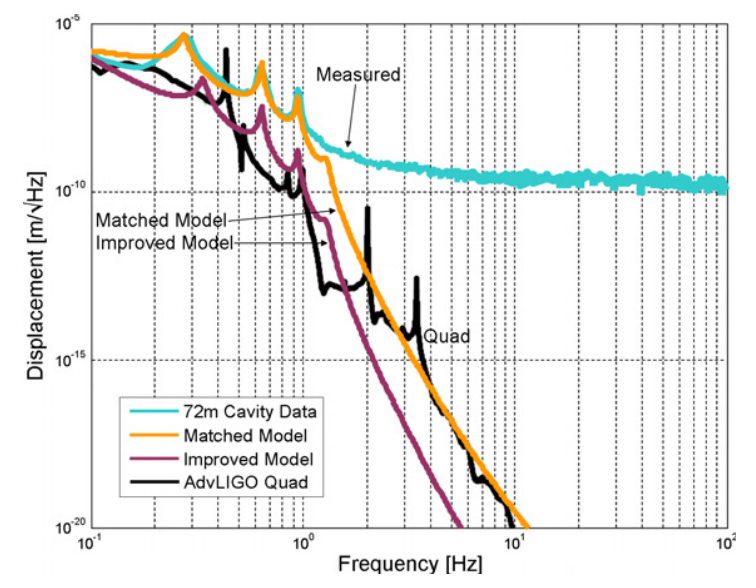

(b)

Figure 7. (a) Full vibration isolator system schematic that shows the different stages of preisolation and the multi-pendulum stage with a test mass at the bottom of the chain. $(b)$ Measured curve shows laser noise above $1 \mathrm{~Hz}$, where the matched model shows the expected behaviour with no laser noise. The improved model shows the expected displacement after the implementation of the super-spring concept. For comparison the expected Advanced LIGO four stage suspension (QUAD) is shown.

\subsection{High power laser}

It is proposed that AIGO will use the same laser system designed for Advanced LIGO and developed by Laser Zentrum Hannover (LZH) and the Albert-Einstein-Institut (Hannover) [40]. It is based on a high stability low power master laser with low frequency fluctuations, which is amplified in a master-oscillator power-amplifier (MOPA) configuration to $35 \mathrm{~W}$. The slave oscillator is an injection-locked oscillator that relies on a birefringence compensation scheme [41].

Up to the medium power stage, the laser corresponds to the MOPA laser currently being used in the Enhanced LIGO science run [42]. After high power amplification a bow-tie configuration pre-mode cleaner is installed in order to reduce higher order optical modes and provide filtering for power noise at radio frequencies. A reference cavity provides frequency stabilization with feedback to the NRPO laser. By controlling the temperature of this reference cavity one can stabilize the laser to the slow drift of the long arm cavities due to Earth tides. An AOM frequency shifter placed in the beam path towards the reference cavity is used as an actuator to stabilize the laser to the AIGO suspended input mode cleaner, which is a much quieter frequency reference.

\subsection{Vacuum system}

Following the successful experience of LIGO the long baselines will be fabricated from pretreated, air-baked 304L-grade stainless steel (SS304 L). The partial pressure of hydrogen is required to be $\sim 10^{-9}$ mbar to obtain residual gas-phase noise of $\sim 10^{-25} \mathrm{~Hz}^{-1 / 2}$ [43]. The vacuum-tube diameter $(\sim 1.2 \mathrm{~m})$ is set by the light-scattering requirements, and also by the possible need for additional optical cavities within the tube, such as a suspension point interferometer [44].

The SS304 L, while in the form of a roll, will be baked at $450{ }^{\circ} \mathrm{C}$ for $36 \mathrm{~h}$ in a low-humidity air atmosphere to reduce the hydrogen outgassing rate [45]. The baffles, designed to minimize 
the scattering of light within the tube, will also undergo baking and will be installed along with the tube fabrication. Once the tube is installed low temperature vacuum baking $\left(150{ }^{\circ} \mathrm{C}\right)$ to remove species such as $\mathrm{H}_{2} \mathrm{O}, \mathrm{CO}$ and $\mathrm{CO}_{2}$ [46-48] will be performed using a solar bakeout technique already tested experimentally [49]. Stiffening rings will be used at regular intervals to prevent the collapse of the tube under atmospheric pressure and every $200 \mathrm{~m}$ bellows will be located to compensate for thermal expansion. The tube will be supported on concrete footings along the length to ensure linearity within $5 \mathrm{~mm}$.

A prototype vacuum control system is working satisfactorily at the $80 \mathrm{~m}$ test facility in Gingin and is planned to be extended to the $4 \mathrm{~km}$ of the full interferometer. To achieve the vacuum specifications it is necessary to use three or four pumps along each arm. End-station tanks will be pumped separately, so they contribute no additional load. The effects of baffles on the ultimate vacuum were modelled by Sunil [50], showing that baffles only degrade the ultimate vacuum by a few per cent.

\section{Conclusion}

We have shown that an advanced GW detector similar in sensitivity to Advanced LIGO and Advanced Virgo makes a major improvement to the existing international network of detectors, greatly increasing the expected science return. AIGO increases the projected average baseline of the global array of ground-based GW detectors by a factor $\sim 4$. The orientation of AIGO allows much better resolution of both wave polarizations. This enables better distance estimates for inspiral events, allowing unambiguous optical identification of host galaxies for about $25 \%$ of neutron star binary inspiral events. This can allow Hubble law estimation without optical identification of an outburst, and can also allow imaging with EM telescopes to search for weak afterglows. This allows independent estimates of cosmological acceleration and dark energy as well as improved understanding of the physics of neutron star and black hole coalescences.

The optical configuration of AIGO is identical to the Advanced LIGO one, except for the possible use of optical fibre mode cleaners. The laser system will utilize an AEI Max-Planck high power pre-stabilized laser. The digital control hardware will follow the Advanced LIGO design. The isolation and suspension design could make use of soft isolation technology developed and recently demonstrated at Gingin. Downselection will be the subject of a review which will be undertaken before completion of the vacuum infrastructure. The strain sensitivity of AIGO will match that of Advanced LIGO. Quantum squeezing technology will be used to provide enhanced sensitivity in the high frequency part of the sensitivity band.

\section{Acknowledgments}

The authors gratefully acknowledge the support of the United States National Science Foundation for the construction and operation of the LIGO Laboratory and the Science and Technology Facilities Council of the United Kingdom, the Max-Planck-Society and the State of Niedersachsen/Germany for support of the construction and operation of the GEO600 detector. The authors also gratefully acknowledge the support of the research by these agencies and by the Australian Research Council, the Department of Science \& Technology, India, under the Science \& Technology Cooperative Programme between India and Australia, Centre National de la Recherche Scientifique, France, the Royal Society, the Scottish Funding Council, the Scottish Universities Physics Alliance and the National Aeronautics and Space Administration. 


\section{References}

[1] Abbott B P et al 2009 Rep. Prog. Phys. 72076901 (25pp)

[2] Acernese F et al 2008 Class. Quantum Grav. 25114045 (8pp)

[3] Grote H (for the LIGO Scientific Collaboration) 2008 Class. Quantum Grav. 25114043 (9pp)

[4] Harry G (for the LIGO Scientific Collaboration) 2010 Class. Quantum Grav. 27084006

[5] Flaminio R et al 2005 Technical Report VIR-NOT-DIR-1390-304 Virgo

[6] Willke B et al 2006 Class. Quantum Grav. 23 S207-14

[7] Kuroda K (for the LCGT Collaboration) 2006 Class. Quantum Grav. 23 S215-21

[8] Wen L et al 2007 Proc. of the XLIInd Rencontres de Moriond on Gravitational Waves and Experimental Gravity (Hanoi: The Gioi Publishers) pp 123-31

[9] Dalal N et al 2006 Phys. Rev. D 74063006

[10] Schutz B F 1986 Nature 323 310-1

[11] Coward D M et al 2009 Publ. Astron. Soc. Aust. submitted

[12] Gürsel Y and Tinto M 1989 Phys. Rev. D 40 3884-3938

[13] Coward D et al 2005 Rev. Sci. Instrum. 76044501

[14] Drever R W P 1983 Lecture Notes in Physics vol 124 (Berlin: Springer) pp 321-38

[15] Arain M A and Mueller G 2008 Opt. Express 16 10018-32

[16] Barriga P et al 2009 Opt. Express 17 2149-65

[17] Rüdiger A et al 1981 J. Mod. Opt. 28 641-58

[18] Rong H et al 2002 9th Marcel Grossmann Meeting pp 1837-8

[19] Barriga P et al 2006 J. Phys. Conf. Ser. 32 457-63

[20] Livas J et al 1986 Fourth Marcel Grossmann Meeting pp 591-7

[21] LaPenna P 2006 Technical Report G070361-00-Z LIGO

[22] Fan Y et al 2008 Rev. Sci. Instrum. 79104501

[23] Harry G M et al 2007 Class. Quantum Grav. 24 405-15

[24] Abbott R et al 2008 Technical Report T070247-00 I LIGO

[25] Miyakawa O et al 2006 J. Phys. Conf. Ser. 32 265-9

[26] Buonanno A, Chen T and Mavalvala N Phys. Rev. D 672003

[27] Fritschel P and Sanders G 2000 Technical Report T000062-00-D LIGO

[28] Hild S et al 2009 Class. Quantum Grav. 26055012

[29] Flaminio R (Virgo Collaboration) 1998 Nucl. Instrum. Methods A 409 477-9

[30] Vahlbruch H, Chelkowski S and Hage B 2006 Class. Quantum Grav. 23 S251-7

[31] Abbott R et al 2004 Class. Quantum Grav. 21 S915-21

[32] Losurdo G et al 1999 Rev. Sci. Instrum. 70 2507-15

[33] Plissi M V et al 2000 Rev. Sci. Instrum. 71 2539-45

[34] Marka S, Takamori A and Ando M 2002 Class. Quantum Grav. 19 1605-14

[35] Rinker R 1983 PhD Thesis Department of Physics, University of Colorado at Boulder

[36] Winterflood J 2001 PhD Thesis School of Physics, The University of Western Australia

[37] Lee B H, Ju L and Blair D G 2006 Phys. Lett. A 350 319-23

[38] Dumas J C et al 2009 Rev. Sci. Instrum. 80114502

[39] Barriga P et al 2009 Rev. Sci. Instrum. 80114501

[40] Willke B et al 2008 Class. Quantum Grav. 25114040

[41] Frede M et al 2005 Opt. Express 13 7516-9

[42] Frede M et al 2007 Opt. Express 15 459-65

[43] Coyne D 2004 Technical Report T040001-00-D LIGO

[44] Aso Y et al 2004 Phys. Lett. A 327 1-8

[45] Carpenter W A et al 2000 J. Vac. Sci. Technol. A 18 1794-9

[46] Odaka K and Ueda S 1995 J. Vac. Sci. Technol. A 13 520-3

[47] Bernardini M et al 1998 J. Vac. Sci. Technol. A 16 188-93

[48] Bennett J R J et al 2004 Vacuum 73 149-53

[49] Berinson D et al 1993 Vacuum 44 151-4

[50] Sunil S and Blair D G 2007 J. Vac. Sci. Technol. A 25 763-8 